\title{
A design model to predict optimal two-material composite structures
}

\author{
H. Rodrigues \\ IDMEC-Instituto Superior Técnico, Av Rovisco Pais, 1096 Lisboa Codex, Portugal \\ hcr@alfa.ist.utl.pt
}

Ciro A. Soto

Ford Research Laboratories, Dearborn, Michigan 48121-2053, USA

csoto@ford.com

\section{J.E. Taylor}

University of Michigan, Ann Arbor, Michigan 48109, USA

janos@engin.umich.edu

\begin{abstract}
A method is presented for the prediction of optimal configurations for two-material composite continuum structures. In the model for this method, both local properties and topology for the stiffer of the two materials are to be predicted. The properties of the second, less stiff material are specified and remain fixed. At the start of the procedure for computational solution, material composition of the structure is represented as a pure mixture of the two materials. This design becomes modified in subsequent steps into a form comprised of a skeleton of concentrated stiffer material, together with a nonoverlapping distribution of the second material to fill the original domain. Computational solutions are presented for two example design problems. A comparison among solutions for different ratios of stiffness between the two materials gives an indication of how the distribution of concentrated stiffer material varies with this factor. An example is presented as well to show how the method can be used to predict an efficient layout for rib-reinforcement of a stamped sheet metal panel.
\end{abstract}

\section{Introduction}

We address the problem of how to predict the optimal configuration of a continuum structure composed of two distinct, linearly elastic materials. The problem is cast in a form that has the stiffer of the component materials treated as "designable", while properties of the second, less-stiff material are taken to be specified and fixed. The goal in the treatment of this design problem is twofold: (i) to determine the local properties of the concentrated stiffer material and (ii) to determine its optimal topology, imbedded in the second material which occupies all the remainder of the original domain of the structure. The intention is that this result simulates an optimally reinforced two-material composite continuum structure. An interpretation of the model for computational solution is applied to produce example results in $2 \mathrm{D}$ and $3 \mathrm{D}$ showing the form of such designs. As another type of application, the modelling technique is applied to design the optimal layout for rib-reinforcement in a stamped sheet metal panel.

The analytical formulation for the composite design problem is based on the concept that has a designable continuum material represented (for linearly elastic material) by its constitutive tensor. The paper by Bendsøe et al. (1994) evidently is the first example where the arbitrary tensor-valued function representing material properties is treated directly as the design variable. [A quite different approach to represent variable material property within a design problem is described by Jacobs et al. (1997)]. In that presentation as well as subsequent other applications (see e.g. Bendsøe et al. 1995, 1996; Bendsøe 1995), the design objective was to minimize compliance and the global cost (isoperimetric) constraint was expressed in terms of the trace or the second-order invariant of the modulus tensor. The present formulation is stated for the same objective, and for the cost constraint based on the trace measure of the modulus tensor.

Results from the cited earlier design formulations are comprised of a prediction of the local form of the optimal material (a zero-shear stiffness, orthotropic material in the case of single-purpose design), together with the (continuously varying) distribution of the trace of the modulus tensor, the latter representing a measure of merit of the material. In the present setting, the corresponding result has the form of an optimal distribution of a pure mixture of the two constituent materials, having effective modulus equal to the sum of the constituent moduli. In order to be able to determine the design for a physically realistic composite material, i.e. a structural composite where the two materials are distinct, a recently described technique [see Guedes and Taylor (1997a,b) and Pawlicki et al. (1998) for example applications in 3D] for the computational prediction of optimal topology is applied. The technique, which differs sharply from the familiar approach [see e.g. Bendsøe (1995) and Olhoff et al. (1997) for one material structures and Olhoff et al. (1993) in the case of two-dimensional structures composed of two materials], amounts to a step by step computational procedure, making 
use of the above described continuously varying design as a starting point. A finite sequence of repeated solutions to the original design problem, each with stepwise, ordered modification to the "unit cost distribution", leads to the final result in the form of a composite having the two materials appear as effectively distinct but mechanically combined. [A distinctly different example where "overlap of materials' is addressed is reported by Rozvany et al. (1982).]

To summarize the contents of the paper, the model for prediction of the optimal composite continuum structure is described first in the form of an algorithm, where the elements described above are identified with steps in the algorithm, as are the means to manage the step-wise procedure itself. Implementation of the procedure into a form suitable for the production of computational results is described next. Example results are presented for the "finite composite design" of a cantilevered beam under end load, and for a clamped beam subject to a distributed load. Sets of results are obtained for both examples to indicate how the layout of the stiffer material (reinforcement) is affected by varying the relative stiffness of the two materials. As indicated earlier, the model for composite design also is applied to predict optimal patterns for rib reinforcement of a sheet metal panel [the interpretation may be contrasted to the treatment of reinforcement of plates by Diaz et al. (1995)], and computational results for an example of this application are presented as well.

\section{Model for the procedure}

Let us consider the linear elastic structure occupying domain $\Omega$, subjected to body forces $\mathbf{f}$, boundary tractions $\mathbf{t}$ and zero displacement on boundary $\Gamma_{u}$. The structure will be composed of two materials identified respectively by their elasticity tensors $\mathbf{E}^{1}$, for the weaker material and $\mathbf{E}^{2}$ for the stiffer material (see Fig. 1a). In a simple fiber-reinforced composite, for example, the two moduli might correspond to the matrix and the fiber materials, respectively.

The problem we address here is the following. Assuming that the domain $\Omega$ occupied by the complete composite structure is specified and fixed, and given an upper bound on the amount of available material "2" (stiffer material), we seek to identify optimal properties and topology of material "2" imbedded in material " 1 ", with no overlap of the less stiff material in the region of the stiffer one (see Fig. 1b). The objective for optimal design is to maximize a measure of the overall stiffness of the structure. The $\mathbf{E}^{1}$ material properties are taken to be specified and fixed with $\mathbf{E}^{1}>0$. With $\Omega_{2}$ to symbolize the part of the domain taken up by concentrated material "2" (to be optimally designed), it is required that in the final design $\Omega_{1}=\Omega-\Omega_{2}$. Since the structural domain $\Omega$ is fixed, this indicates that material " 1 " fills the part of the original domain not occupied by the optimal material " 2 " without overlap.

In order to solve the problem described above, the following procedure is proposed.

Initial design. As a first step assume that one has a perfect mixture of the two materials [i.e. the effective material tensor of the mixture is given by $\mathbf{E}^{\text {eff }}=\mathbf{E}^{1}+\mathbf{E}^{2}$; note that for the $2 \mathrm{D}$ model to simulate laminated structure (see e.g.

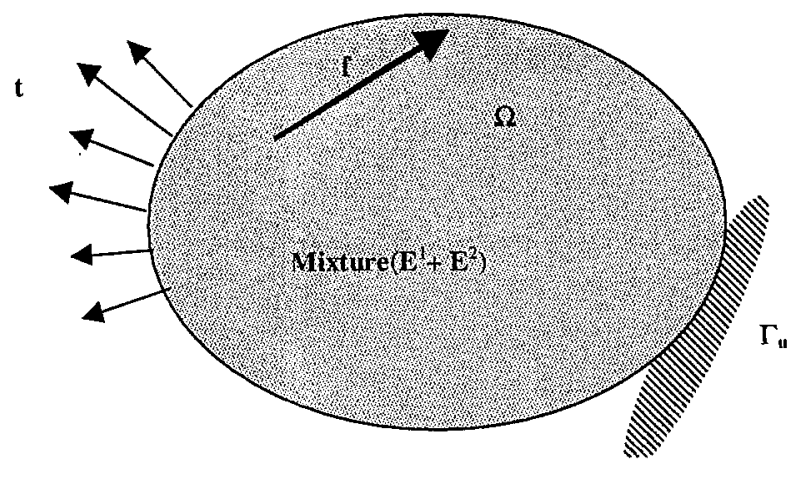

a)

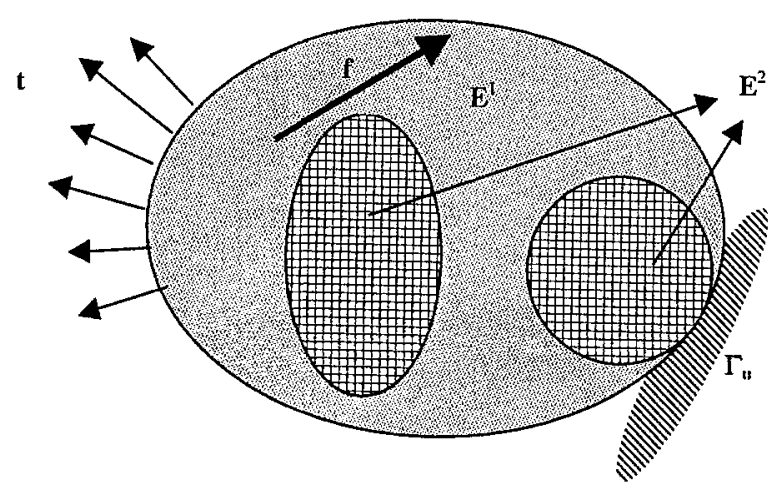

b)

Fig. 1. The structure subjected to body force $\mathbf{f}$ and boundary traction t. (a) Initially as a perfect mixture, and (b) as a two material composite

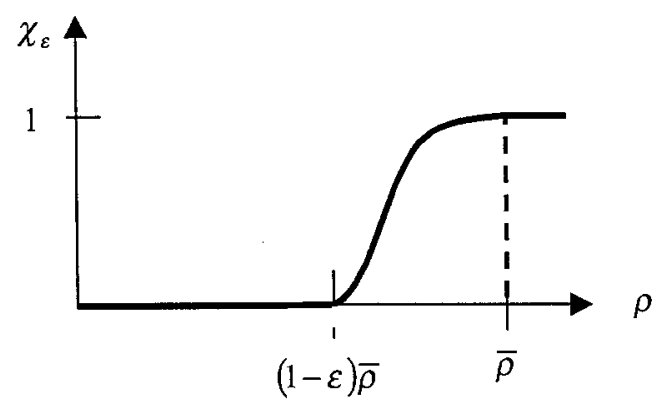

Fig. 2. Regularization of the characteristic function

Pedersen, 1993) the "mixture" provides an authentic model for the effective material modulus]. Again, recall that at the final design the materials are to be effectively separated.

For this mixture and identifying the trace of the $\mathbf{E}^{2}$ tensor as the sensible measure of the material $\left[\rho=\operatorname{tr}\left(\mathbf{E}^{2}\right)\right]$, design material " 2 " to obtain its optimal local properties and distribution in $\Omega$. Here the resulting design, obtained using the method described by Bendsøe et al. (1994), is represented by continuously varying $\rho$ (the plot of $\rho$ is sometimes referred to as the "shades of grey" diagram). Once this solution for 
the optimal material and its distribution over the structure are known, a finite number of optimization sub-problems are performed to predict a refinement of it into a design having total material separation and concentration of material " 2 " at its upper bound. The redesign of material " 2 " is accomplished using a weighted unit relative cost method (Guedes and Taylor 1997a) for the prediction of optimal topology. The method is applied stepwise, where in each step a gradually higher value of unit cost is ascribed to regions of relatively low value of $\rho$. Specific details are provided next, where the procedure is first described formally in the form of an algorithm, and is later expressed for computational treatment using a finite element interpretation of the continuum structure.

\subsection{Algorithm}

It is assumed that an initial design (the shades of grey results described above) has been obtained. Based on this result and recalling that "design" is represented by the $\rho$ field, the algorithm is described as follows.

Step 1. Define an increasing sequence of $N$ evenly spaced cutoff values $\rho_{k}^{c}, k=1,2, \ldots, N$ such that $\rho_{1}^{c}=\bar{\rho} / N$ and $\rho_{n}^{c}=\bar{\rho} ; \rho_{k}^{c}=k(\bar{\rho} / N)$. An optimization subproblem is to be solved for each value of $\rho_{k}^{c}$, where index $k$ identifies steps in the procedure. The number $N$ designates the number of steps to achieve the final design. Both $N$ and the upper bound $\bar{\rho}$ are prescribed. Considerations of how properly to select values of these data are discussed below.

Step 2 For a given cut off value $\rho_{k}^{c}$, identify the following subdomains of the structure:

$\Omega_{k}^{+}=\left\{\mathbf{x} \in \Omega: \rho_{k} \geq \rho_{k}^{c}\right\}$,

subdomain with higher value of material measure $\rho$,

$\Omega_{k}^{-}=\left\{\mathbf{x} \in \Omega: \rho_{k}<\rho_{k}^{c}\right\}$,

subdomain with lower value of material measure $\rho$,

$\bar{\Omega}_{k}=\left\{\mathbf{x} \in \Omega: \rho_{k}=\bar{\rho}\right\}$,

subdomain with material measure $\rho$ at its upper bound.

Recall that $\rho$ is identified as the trace measure of the elasticity tensor $\mathbf{E}^{2}$, and note that $\bar{\Omega}_{k} \subset \Omega_{k}^{+}$ and $\Omega_{k}^{+} \cup \Omega_{k}^{-}=\Omega$.

Step 3 . With the objective of driving the designable material at each point to either its upper $(\bar{\rho})$ or lower $(\rho)$ bound value, a unit relative cost coefficient $\omega$ is modified stepwise in a manner to exaggerate the cost of material wherever $\rho$ has value below the cutoff. Specifically, the relative unit cost function based on the results at step $k$ is set equal to the constant value one or $\bar{\omega}$ according to

$\omega_{k}=\left\{\begin{array}{l}1 \text { for } \mathbf{x} \in \Omega_{k}^{+} \\ \bar{\omega} \text { for } \mathbf{x} \in \Omega_{k}^{-}\end{array}\right.$, with value of the constant $\bar{\omega} \gg 1$. Total cost at the $(k+1)$-st step is evaluated according to

$$
\int_{\Omega} \omega_{k} \rho_{k+1} \mathrm{~d} \Omega
$$

Step 4. Once the relative unit cost is defined, optimize the material "2" elasticity tensor $\mathbf{E}^{2}$, i.e. determine the measure $\rho_{k+1}$, and the remaining attributes of $\mathbf{E}^{2}$ using the cost distribution determined in Step 3 . This is accomplished by solving the (shades of grey) problem,

$$
\min _{\substack{\mathbf{E}^{2} \\ 0<\rho \leq \rho_{k+1} \\ \operatorname{tr}\left(\mathbf{E}^{2}\right) \leq \bar{\rho}}}\left[\int_{\Omega} f_{i} u_{i} \mathrm{~d} \Omega+\int_{\Gamma} t_{i} u_{i} \mathrm{~d} \Gamma\right],
$$

subjected to the following constraints:

$$
\begin{aligned}
& \int_{\Omega}\left[1-\chi\left(\bar{\Omega}_{k}\right)\right] E_{i j k \ell}^{1} e_{i j}(\mathbf{u}) e_{k \ell}(\mathbf{v}) \mathrm{d} \Omega+ \\
& \int_{\Omega} E_{i j k \ell}^{2} e_{i j}(\mathbf{u}) e_{k \ell}(\mathbf{v}) \mathrm{d} \Omega-\int_{\Omega} f_{i} v_{i} \mathrm{~d} \Omega- \\
& \int_{\Gamma_{t}} t_{i} v_{i} \mathrm{~d} \Gamma=0, \quad \forall \mathbf{v} \in \mathbf{V}, \\
& \int_{\Omega_{k}^{+}} \rho_{k+1} \mathrm{~d} \Omega+\int_{\Omega_{k}^{-}} \bar{\omega} \rho_{k+1} \mathrm{~d} \Omega \leq R,
\end{aligned}
$$

where $\mathbf{V}$ represents the set of admissible displacements and where $\chi\left(\bar{\Omega}_{k}\right)$ is the characteristic function defined in terms of the set $\bar{\Omega}_{k}$ as

$\chi\left(\bar{\Omega}_{k}\right)=\left\{\begin{array}{l}1 \text { for } x \in \bar{\Omega}_{k} \\ 0 \text { for } x \notin \bar{\Omega}_{k}\end{array}\right.$.

Note that $\mathbf{u}$ and $\mathbf{E}^{2}$ in (7) are intended to stand for the solution of the current step.

An alternative, equivalent statement of the equilibrium condition (7) is

$$
\begin{aligned}
& \int_{\Omega-\bar{\Omega}_{k}}\left(E_{i j k \ell}^{1}+E_{i j k \ell}^{2}\right) e_{i j}(\mathbf{u}) e_{k \ell}(\mathbf{v}) \mathrm{d} \Omega+ \\
& \int_{\bar{\Omega}_{k}} E_{i j k \ell}^{2} e_{i j}(\mathbf{u}) e_{k \ell}(\mathbf{v}) \mathrm{d} \Omega-
\end{aligned}
$$$$
\int_{\Omega} f_{i} v_{i} \mathrm{~d} \Omega-\int_{\Gamma_{i}} t_{i} v_{i} \mathrm{~d} \Gamma=0, \quad \forall \mathbf{v} \in \mathbf{V}
$$ 
Note that in form (10) for the problem, separation of the structure in $\Omega$ into the domain $\bar{\Omega}_{k}$ where $\rho(\mathbf{x})=$ $\bar{\rho}$ and the remaining $\Omega-\bar{\Omega}_{k}$ is reflected explicitly in the integrals.

The lower bound $\rho$ is set to have the value on the order $10^{-7} \bar{\rho}$.

For the equilibrium constraint (7) note that the fixed material "1" exists (mixed with material "2") only where the measure of material "2" is not its upper bound, i.e. material " 1 " is removed from the region with full material "2". As the procedure converges this will provide full pointwise separation of the two materials. Also in the resource constraint we assume, as previously stated, that the material unit cost for higher value of the material measure $\rho$ is equal to one, and for the lower value of the measure is $\bar{\omega} \gg 1$. The distribution of material " 1 " is fixed during this step, and the solution obtained provides the description $\rho_{k+1}$ of the trace of $\left(\mathbf{E}^{2}\right)_{k+1}$ as well as the current local material properties $\left(E_{i j k \ell}^{2}\right)_{k+1}$.

Step 5. Set the cut off value to $\rho_{k+1}^{c}$.

Go to Step 2 and repeat the procedure until the limiting cutoff value $\bar{\rho}$ is reached.

\subsection{Optimal distribution of the designable material}

We will analyse in more detail the optimization subproblem to be solved in Step 4 of the previous section. The aim of this step is to solve the following problem. For a prescribed material unit cost function $\omega(x)$ and fixed sets $\bar{\Omega}_{k}, \Omega_{k}^{+}$and $\Omega_{k}^{-}$, find the optimal distribution of designable material as the one that minimizes the structural compliance (maximizes the stiffness) of the structure, as stated in the optimization problem (6)-(8).

Following the presentation in the cited Bendsøe et al. (1994) paper for the treatment of this problem, the optimization problem can be equivalently stated as

$$
\begin{aligned}
& \max _{\substack{\mathbf{E}^{2} \geq 0 \\
\rho \leq \rho=\operatorname{tr}\left(\mathbf{E}^{2}\right) \leq \bar{\rho}}} \min _{\mathbf{v} \in \mathbf{V}}\left\{\frac { 1 } { 2 } \left\{\int_{\Omega}\left[1-\chi\left(\bar{\Omega}_{k}\right)\right] \times\right.\right. \\
& \left.E_{i j k \ell}^{1} e_{i j}(\mathbf{v}) e_{k \ell}(\mathbf{v}) \mathrm{d} \Omega+\int_{\Omega} E_{i j k \ell}^{2} e_{i j}(\mathbf{v}) e_{k \ell}(\mathbf{v}) \mathrm{d} \Omega\right\}- \\
& \left.\int_{\Omega} f_{i} v_{i} \mathrm{~d} \Omega-\int_{\Gamma_{t}} t_{i} v_{i} \mathrm{~d} \Gamma\right\}
\end{aligned}
$$

subjected to the resource constraint

$$
\int_{\Omega_{k}^{+}} \operatorname{tr}\left(\mathbf{E}^{2}\right) \mathrm{d} \Omega+\int_{\Omega_{k}^{-}} \bar{\omega} \operatorname{tr}\left(\mathbf{E}^{2}\right) \mathrm{d} \Omega \leq R .
$$

Note that in this statement of the structural compliance optimization problem, the equilibrium constraint is interpreted in the form of a minimum potential energy statement.
The design variable $\mathbf{E}^{2}$ is characterized by the field $\rho(x)$ which identifies global distribution of the trace measure of $\mathbf{E}^{2}$, and by its local tensorial structure. The maximization problem in (11) can be split into two successive maximization problems (see Bendsøe et al. 1994), associated respectively with these "field" and "local structure" attributes of $\mathbf{E}^{2}$.

Thus (11) is rewritten as

$$
\begin{aligned}
& \max _{\substack{\left.\rho \leq \rho \leq \bar{\rho} \\
\max _{\operatorname{tr}\left(\mathbf{E}^{2}\right)=\rho} \mathbf{E}^{2}\right)=\rho}} \min _{\mathbf{v} \in \mathbf{V}}\left\{\frac { 1 } { 2 } \left\{\int_{\Omega}\left[1-\chi\left(\bar{\Omega}_{k}\right)\right] \times\right.\right. \\
& \left.E_{i j k \ell}^{1} e_{i j}(\mathbf{v}) e_{k \ell}(\mathbf{v}) \mathrm{d} \Omega+\int_{\Omega} E_{i j k \ell}^{2} e_{i j}(\mathbf{v}) e_{k \ell}(\mathbf{v}) \mathrm{d} \Omega\right\}- \\
& \left.\int_{\Omega} f_{i} v_{i} \mathrm{~d} \Omega-\int_{\Gamma_{t}} t_{i} v_{i} \mathrm{~d} \Gamma\right\},
\end{aligned}
$$

where the inner maximization identifies the optimal elasticity tensor within a prescribed value of the trace $\rho$ (optimal relative values among tensor components) and the outer max establishes the optimal distribution of the trace of the elasticity tensor within the prescribed upper and lower bounds (optimal material distribution).

From the fact that the resource constraint is only dependent on the design variable $\rho$, i.e. on a norm of the tensor $\mathbf{E}^{2}$ (the trace in our case) and not on its individual components, consider, for now, only the inner max min problem in (13). Following the same argument as presented by Bendsøe et al. (1994), the inner max and min can be interchanged as

$$
\begin{aligned}
& \min _{\mathbf{v} \in \mathbf{V}} \max _{\substack{\mathbf{E}^{2} \geq 0 \\
\operatorname{tr}\left(\mathbf{E}^{2}\right)=\rho}}\left\{\frac { 1 } { 2 } \left\{\int_{\Omega}\left\{1-\chi\left(\bar{\Omega}_{k}\right)\right] \times\right.\right. \\
& \left.E_{i j k \ell}^{1} e_{i j}(\mathbf{v}) e_{k \ell}(\mathbf{v}) \mathrm{d} \Omega+\int_{\Omega} E_{i j k \ell}^{2} e_{i j}(\mathbf{v}) e_{k \ell}(\mathbf{v}) \mathrm{d} \Omega\right\}-
\end{aligned}
$$

$$
\left.\int_{\Omega} f_{i} v_{i} \mathrm{~d} \Omega-\int_{\Gamma_{t}} t_{i} v_{i} \mathrm{~d} \Gamma\right\}
$$

Following this transformation, the maximum can be solved analytically for the design variable $\mathbf{E}^{2}$ and has the solution

$E_{i j k \ell}^{2^{*}}=\rho \frac{e_{i j}(\mathbf{v}) e_{k \ell}(\mathbf{v})}{\left\|e_{i j}(\mathbf{v})\right\|^{2}}$

with the optimal strain energy density equal to

$\frac{1}{2} E_{i j k \ell}^{2^{*}} e_{i j}(\mathbf{v}) e_{k \ell}(\mathbf{v})=\frac{1}{2} \rho e_{i j}(\mathbf{v}) e_{i j}(\mathbf{v})$.

Substituting (16) into (13) one obtains

$\max _{\underline{\rho} \leq \rho \leq \bar{\rho} \mathbf{v} \in \mathbf{V}}\left\{\frac{1}{2}\left\{\int_{\Omega}\left[1-\chi\left(\bar{\Omega}_{k}\right)\right] \times\right.\right.$ 


$$
\left.E_{i j k \ell}^{1} e_{i j}(\mathbf{v}) e_{k \ell}(\mathbf{v}) \mathrm{d} \Omega+\int_{\Omega} \rho \delta_{i k} \delta j \ell e_{i j}(\mathbf{v}) e_{k \ell}(\mathbf{v}) \mathrm{d} \Omega\right\}-
$$

$\left.\int_{\Omega} f_{i} v_{i} \mathrm{~d} \Omega-\int_{\Gamma_{t}} t_{i} v_{i} \mathrm{~d} \Gamma\right\}$

subjected to the resource constraint

$$
\int_{\Omega_{k}^{+}} \rho \mathrm{d} \Omega+\int_{\Omega_{k}^{-}} \bar{\omega} \rho \mathrm{d} \Omega \leq R .
$$

[Note. Result (15) is valid only for one load condition.]

The previous problem is a maxmin problem where the inner minimization has a unique solution. Thus the problem is differentiable and the necessary conditions for a maximum are

$\frac{1}{2} e_{i j}(\mathbf{u}) e_{i j}(\mathbf{u})-\Lambda-\eta_{u}=0, \quad \forall x \in \Omega_{k}^{+}$,

$\frac{1}{2} e_{i j}(\mathbf{u}) e_{i j}(\mathbf{u})-\bar{\omega} \Lambda+\eta_{\ell}=0, \quad \forall x \in \Omega_{k}^{-}$,

where $\mathbf{u}(x)$ is the unique solution of the inner minimization, i.e. the displacement of the structure at equilibrium. Note that these conditions are to be satisfied at the optimum.

In the previous conditions $A$ is the Lagrange multiplier for the resource constraint and $\eta_{u}$ and $\eta_{\ell}$ are the multipliers for the upper and lower bound constraints respectively.

Complementarity conditions for the problem are

$\eta_{u}(\rho-\bar{\rho})=0, \quad \eta_{u} \geq 0, \quad \rho \leq \bar{\rho}, \quad \forall x \in \Omega$,

$\eta_{\ell}(\underline{\rho}-\rho)=0, \quad \eta_{\ell} \geq 0, \quad \rho \geq \underline{\rho}, \quad \forall x \in \Omega$,

$\Lambda\left(\int_{\Omega_{k}^{+}} \rho \mathrm{d} \Omega+\int_{\Omega_{k}^{-}} \bar{\omega} \rho \mathrm{d} \Omega-R\right)=0, \quad \Lambda \geq 0$.

It follows from (21) and (22) that

$\eta_{u} \eta_{\ell}=0, \quad \forall x \in \Omega$.

From the preceding conditions it can be shown that $\Lambda>0$, implying that the resource constraint will be active.

\subsection{Optimal distribution of the designable material (alter- native formulation)}

As an alternative to the part of the procedure of Section 2.1 where the fixed material is removed, it is possible to reformulate the problem of optimal distribution of material so that the fixed material is gradually removed within the process of optimizing the material distribution.

With this purpose in mind, it is assumed that within the material distribution optimization, the domain occupied by the fixed material " 1 " is the complement of the domain
$\bar{\Omega}(\rho)=\{\mathbf{x}: \rho=\bar{\rho}\}$, and consequently a function of $\rho$. Thus problem (13) is restated as

$$
\begin{aligned}
& \max _{0 \leq \rho \leq \bar{\rho}} \max _{\substack{\mathbf{E}^{2} \geq 0 \\
\operatorname{tr}\left(\mathbf{E}^{2}\right)=\rho}} \min _{\mathbf{v} \in \mathrm{V}}\left\{\frac { 1 } { 2 } \left\{\int_{\Omega}[1-\chi \varepsilon(\bar{\Omega}(\rho))] \times\right.\right. \\
& \left.E_{i j k \ell}^{1} e_{i j}(\mathbf{v}) e_{k \ell}(\mathbf{v}) \mathrm{d} \Omega+\int_{\Omega} E_{i j k \ell}^{2} e_{i j}(\mathbf{v}) e_{k \ell}(\mathbf{v}) \mathrm{d} \Omega\right\}-
\end{aligned}
$$

$\left.\int_{\Omega} f_{i} v_{i} \mathrm{~d} \Omega-\int_{\Gamma_{t}} t_{i} v_{i} \mathrm{~d} \Gamma\right\}$,

where the $\chi_{\varepsilon}[\bar{\Omega}(\rho)]$ is an $\varepsilon$ differentiable regularization of the characteristic function of the set $\bar{\Omega}(\rho)=\{\mathbf{x} \in \Omega: \rho=\bar{\rho}\}$, having the properties (see Fig. 2)

$\chi_{\varepsilon}(\rho)= \begin{cases}1 & \text { if } \rho=\bar{\rho} \\ \frac{(\rho+\varepsilon \bar{\rho}-\bar{\rho})^{2}(\varepsilon \bar{\rho}+2 \bar{\rho}-2 \rho)}{(\bar{\rho} \varepsilon)^{3}} & \text { if }(1-\varepsilon) \bar{\rho}<\rho<\bar{\rho} \\ 0 & \text { if } \rho \leq(1-\varepsilon) \bar{\rho}\end{cases}$

Note that this regularization is nonsymmetric in the $\varepsilon$ neighbourhood of $\bar{\varepsilon}$ and thus imposes the effective separation of the two materials on the region $\bar{\Omega}(\rho)$ (i.e. at all the points where $\rho=\bar{\rho}$ ).

Following the rationale of the previous section we will have the same characterization of the optimal material $\mathbf{E}^{2}$ (15), but the necessary conditions on $\rho$ (written for the entire domain) are

$$
\begin{aligned}
& -\frac{\mathrm{d} \chi_{\varepsilon}(\rho)}{\mathrm{d} \rho} \mid E_{i j k \ell}^{1} e_{i j}(\mathbf{u}) e_{k \ell}(\mathbf{u})+e_{i j}(\mathbf{u}) e_{i j}(\mathbf{u})- \\
& \Lambda-\eta_{u}+\eta_{\ell}=0, \quad \forall x \in \Omega,
\end{aligned}
$$

where, $\Lambda, \eta_{u}$ and $\eta_{\ell}$ satisfy the orthogonality conditions (21)(24) and

$$
\frac{\mathrm{d} \chi_{\varepsilon}(\rho)}{\mathrm{d} \rho}=\left\{\begin{array}{ll}
0 & \text { if } \rho=\bar{\rho} \\
\frac{6(\rho-\bar{\rho}+\varepsilon \bar{\rho})(\bar{\rho}-\rho)}{(\bar{\rho} \varepsilon)^{3}} & \text { if }(1-\varepsilon) \bar{\rho}<\rho<\bar{\rho} \\
0 & \text { if } \rho \leq(1-\varepsilon) \bar{\rho}
\end{array} .\right.
$$

The purpose of this regularization is to implicitly remove the fixed material $\mathbf{E}^{1}$ in regions within $\Omega$ where the designable material is at its upper bound. Note that for every $\varepsilon \neq 0$ the problem as stated in (25) is a meaningful design problem. However, the limit case (i.e. when $\varepsilon=0$ ) is not a well-posed problem, in the sense that one can get as close as one wants to the designable material upper bound and still have fixed material coexisting with the designable material.

\section{Method for computational solution}

As described above, the final structural topology is predicted through an iterative procedure where the designable material is concentrated at its upper bound $\bar{\rho}$ (remember that the trace of elastic tensor is identified as such a measure). This is attained through the solution of a series of material optimization subproblems (defined in Section 2.2) where the material unit cost is iteratively adjusted so that lower trace measures impose a higher cost on the resource distribution.

In the following, the implementation of these steps for computation is discussed. 


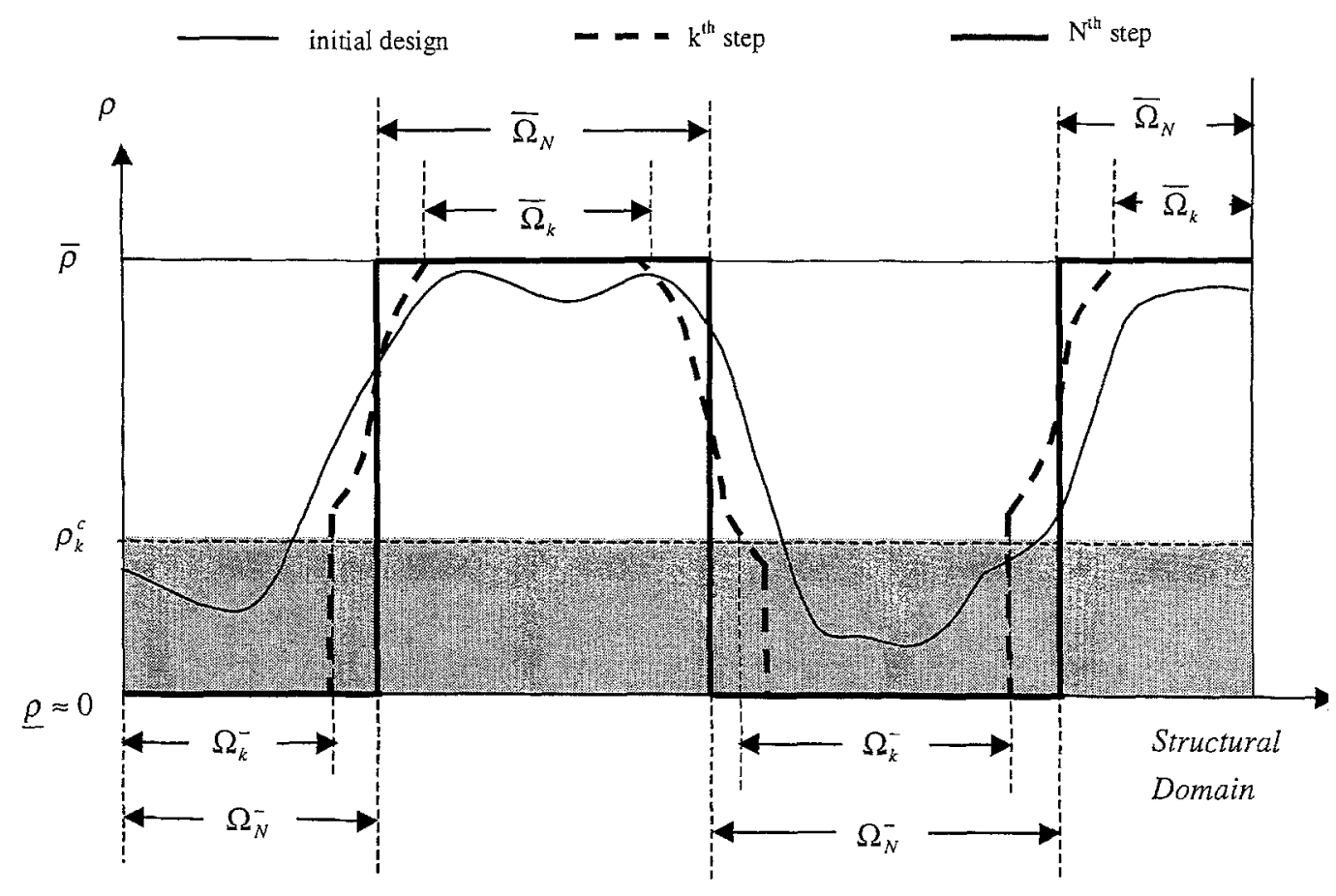

Fig. 3. Sketch of material measure $\rho(\mathbf{x})$ for initial, intermediate and final designs (typical)

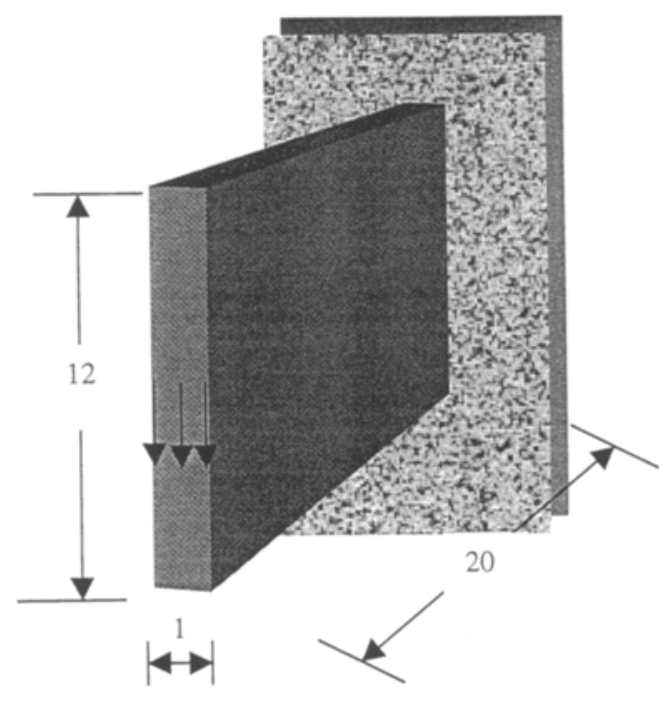

Fig. 4. Design domain and boundary conditions

\subsection{Material unit cost}

The basic idea behind a nonuniform material unit cost imposed on the resource constraint is to induce a concentration of the designable material at its upper bound (full material). As the first step, the optimization problem is solved initially assuming a uniform material unit cost. Based on results for this optimal solution, the structural domain is divided into two regions, the part $\Omega^{-}$(below cutoff value $\rho^{c}$ ) and $\Omega^{+}$ (the part above $\rho^{c}$ ), as defined earlier. Once these sets are defined, one computes the new optimal material distribution imposing a higher unit cost on material in $\Omega^{-}$, i.e. the resource constraint is now changed to

$$
\int_{\Omega^{+}} \rho \mathrm{d} \Omega+\int_{\Omega^{-}} \bar{\omega} \rho \mathrm{d} \Omega \leq R, \quad \bar{\omega} \gg 1 .
$$

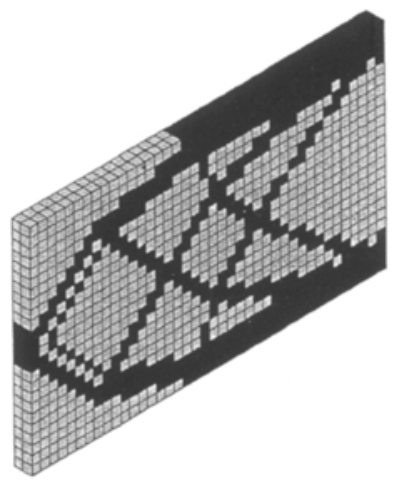

a)

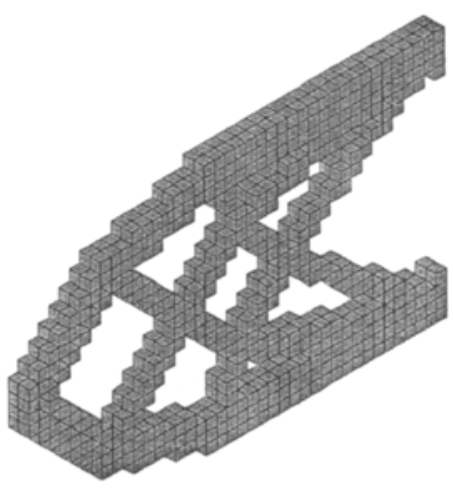

b)
Fig. 5. Optimal topology for $E^{1} /\left(\max E^{2}\right)=10^{-7}$ a)

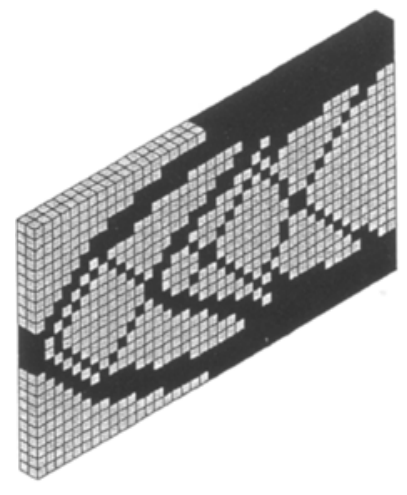

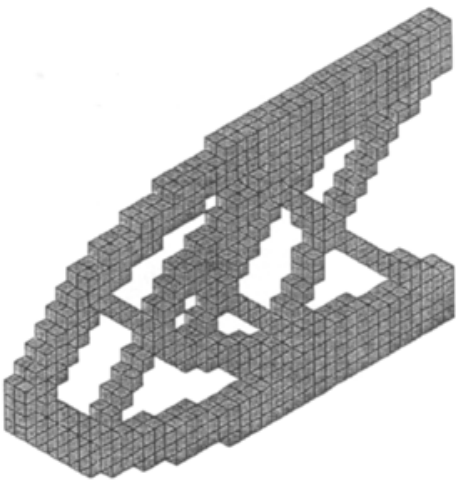

b)
Fig. 6. Optimal topology for $E^{1} /\left(\max E^{2}\right)=1 / 50$

In Fig. 3 this process is simulated in a one-dimensional sketch for initial, intermediate and final distributions. As- 


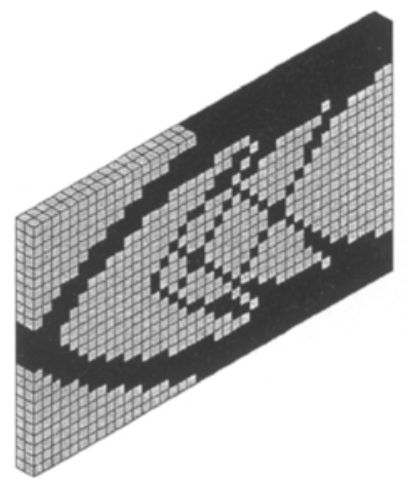

a)

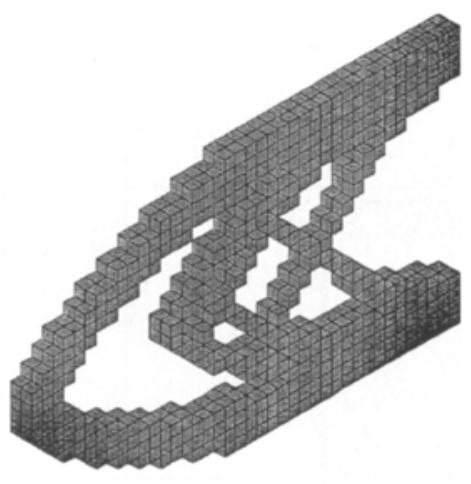

b)
Fig. 7. Optimal topology for $E^{1} /\left(\max E^{2}\right)=1 / 10$

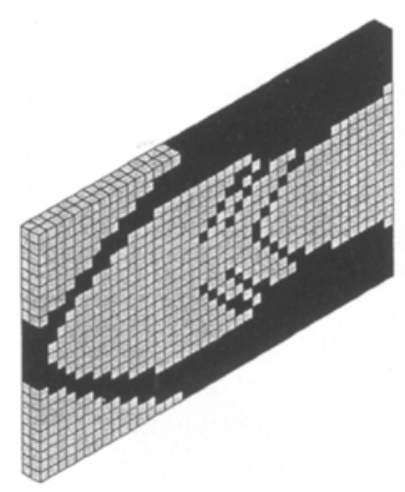

a)

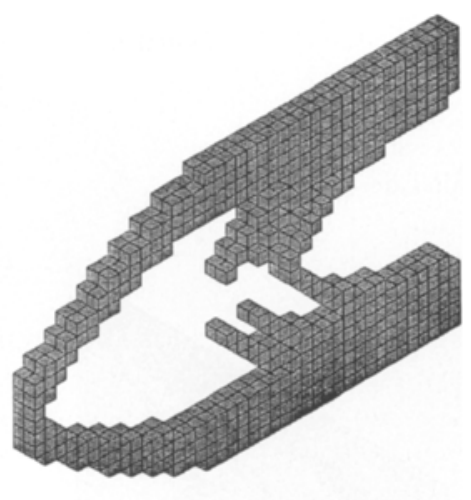

b)
Fig. 8. Optimal topology for $E^{1} /\left(\max E^{2}\right)=1 / 3$

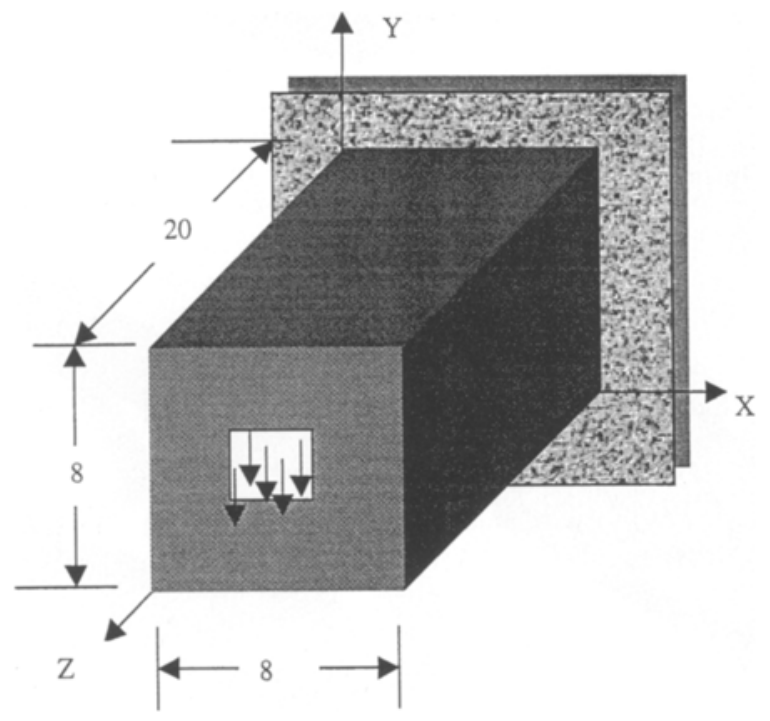

Fig. 9. Design domain and boundary conditions

suming that the material distribution is known for the initial design, the resulting distribution $\rho(\mathbf{x})$ for the subsequent steps (general step $k$ and final step $N$ ) reflects a relative increase value in regions $\left(\Omega-\Omega^{-}\right)$. The increase for the $k$-th step, and the concentration of material at the upper bound for the final design are shown in separate curves.

The idea behind this approach is simple, but one major feature of the method needs to be addressed. It follows from the fact that the procedure attributes higher unit costs according to regions occupied by low material measure $\rho$, rather than to the material itself. As a consequence, once a region is included in the $\Omega^{-}$subdomain it tends to remain there. To deal with such a feature, care should be taken in the selection of the number of cutoff values.

\subsection{Optimal material distribution}

Once the material unit cost function ( $\omega_{k}$ at the $k$-th step of the topology design procedure) is defined, one can calculate the new optimal material distribution. Note that the domain occupied by the fixed material is known and fixed during this step.

The methodology adopted is based on a sequential solution of the optimality conditions (19) and (20) with the strain field calculated using a finite element approximation of the elastostatic problem, characterized by the equilibrium statement (7) (the commercial program ANSYS was exploited for this purpose).

So based on the material unit cost for the procedure step $k\left(\omega_{k}\right)$ and assuming the design variable $\rho^{e}$ constant at each finite element, one can write the optimality conditions separately for each element. From the discrete interpretation of the these conditions, and introducing the upper and lower bound constraint thickness parameter $\xi$ (defined by the user), the solution is obtained by the fixed point method,

$\left(\rho_{e}\right)_{i+1}=\left\{\begin{array}{ll}\frac{M}{\alpha^{e}}\left(\rho^{e}\right)_{i} & \text { if } \alpha^{e}\left(\rho^{e}\right)_{i} \leq M \\ \bar{M} & \left.\text { if } \bar{M} \leq \alpha^{e}\left(\rho^{e}\right)_{i} \leq \bar{M}, \alpha^{e}\right)_{i}\end{array}\right.$,

where

$\underline{M}=\max \left[(1-\xi)\left(\rho^{e}\right)_{i}, \underline{\rho}\right]$,

$\bar{M}=\min \left[(1+\xi)\left(\rho^{e}\right)_{i}, \bar{\rho}\right]$,

and with the multiplier $\alpha^{e}$ given by

$\alpha^{e}=\frac{\left\langle e_{i j}(\mathbf{u}) e_{i j}(\mathbf{u})\right\rangle_{e}}{2 \omega_{k} \Lambda}$

for each finite element.

In the previous algorithm, the index " $e$ " ranges over all the finite elements, " $i$ " is the iteration counter and \langle\rangle$_{e}$ identifies the average operator applied to the $e$-th element strain field.

The calculation of the Lagrange multipliers $\Lambda$ is determined, within each iteration, through a updating scheme that imposes the resource constraint

$\left(\int_{\Omega_{\bar{k}}^{-}} \bar{\omega} \rho \mathrm{d} \Omega+\int_{\Omega_{k}^{+}} \rho \mathrm{d} \Omega\right)=R$.

In the case of the alternative formulation described in Section 2.3 , where the domain occupied with fixed material is 

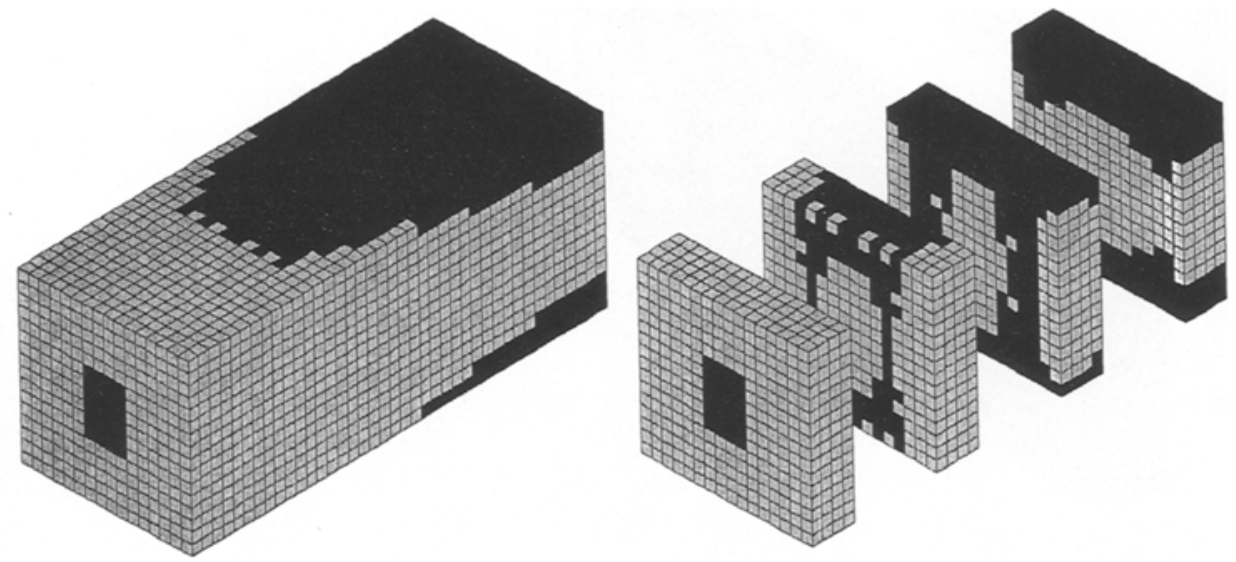

Fig. 10. Final topology for $E^{1} /\left(\max E^{2}\right)=10^{-7}$

gradually removed, the fixed point algorithm is as previously stated in (30) with the $\alpha^{e}$ factor given by

$\alpha^{e}=\frac{\left\langle e_{i j}(\mathbf{u}) e_{i j}(\mathbf{u})\right\rangle_{e}}{2\left[\omega_{k} \Lambda+\frac{\mathrm{d} \chi_{\varepsilon}}{\mathrm{d} \rho} E_{i j k \ell}^{1}\left\langle e_{i j}(\mathbf{u}) e_{i j}(\mathbf{u})\right\rangle_{e}\right]}$.

\section{Examples}

The examples presented below try to demonstrate the applicability of the developments described within the context of three-dimensional structural applications. The first two examples are discretized, for computational purposes with eight-node isoparametric brick elements, a uniform finite element mesh and the computational procedure is programmed within the ANSYS finite element software.

\subsection{Example 1}

The first example considered is the problem of finding the optimal topology of material " 2 " in an end loaded cantilever beam made of an isotropic material with Young's modulus $E^{1}$ and Poisson's coefficient 0.2 (material " 1 "). The design domain has dimensions $20 \times 12 \times 1$ (see Fig. 4) and the resource constraint upper bound equals $40 \%$ of the volume of the design domain.

Material two has a Young's modulus upper bound of $10^{9} \mathrm{~Pa}$ and a lower bound of $10^{1} \mathrm{~Pa}$. The black and white procedure takes $N=40$ steps and the shades of grey optimization (step 4 of the procedure) is limited to 5 iterations.

This example is the $3 \mathrm{D}$ equivalent of the $2 \mathrm{D}$ example described by Guedes and Taylor (1997a). Even though threedimensional, the model would behave like a two-dimensional one due to the small design domain thickness. This fact will permit the comparison of the results obtained with the ones presented for $2 \mathrm{D}$ solutions in the case of nonexistent material "1" (see e.g. Guedes and Taylor 1997a).

The solutions obtained are shown for various ratios between the maximum Young's modulus for material " 2 " ( $\max$ $E^{2}$, attained at $\rho=\bar{\rho}$ ) and the Young's modulus for the fixed material, $E^{1}$.
The first solution has a small value of this ratio $\left(10^{-7}\right)$ and consequently is equivalent to the optimal topology design problem restricted to one material. The subsequent results consider higher material ratios.

Figures on the left show the designable material imbedded within the fixed material. In the right, and to better portray the final topology of the designable material, the fixed material is not shown. Note also that for representation purposes the designable material (material "2") is identified in black color when the two materials are present and in lighter grey when material " 1 " is removed from the figure.

From the results obtained one can observe that the truss like structure inside the domain weakens as the material ratio increases. This is attributed to the fact that as material "1" is stiffened, it has an higher contribution in carrying the shear load present, thus freeing material " 2 " to support the bending load.

\subsection{Example 2}

This example considers the topology optimization of material " 2 " for the cantilever beam presented in Fig. 9. The design domain has dimensions $8 \times 8 \times 20$ and is discretized with 10264 eight-node isoparametric brick elements. The applied force is restricted to a shear force distributed as shown below. The resource constraint upper bound is equal to $40 \%$ of the design domain volume. Material "2" has a Young's modulus upper bound of $10^{9}$ and a lower bound of $10^{1}$. The black and white procedure was done in $N=20$ steps and the shades of grey optimization (Step 4 of the procedure) is limited to five iterations.

The figures below display the results obtained for different ratios of $E^{1} /\left(\max E^{2}\right)$. For this example, the fixed material ("1") is assumed isotropic. For each material ratio, the final result internal structure can be visualized in the various transversal and longitudinal cuts shown.

For this last material ratio, the next figure shows the final topology of material " 2 " with material "1" removed.

Note. For representation purposes the designable material (material "2") is identified in black colour when the two materials are shown and in lighter grey when material " 1 " is 


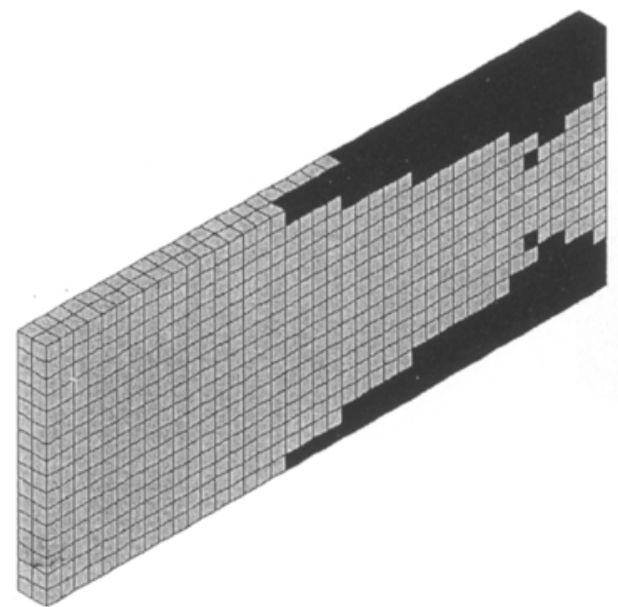

$\mathrm{X}=1$

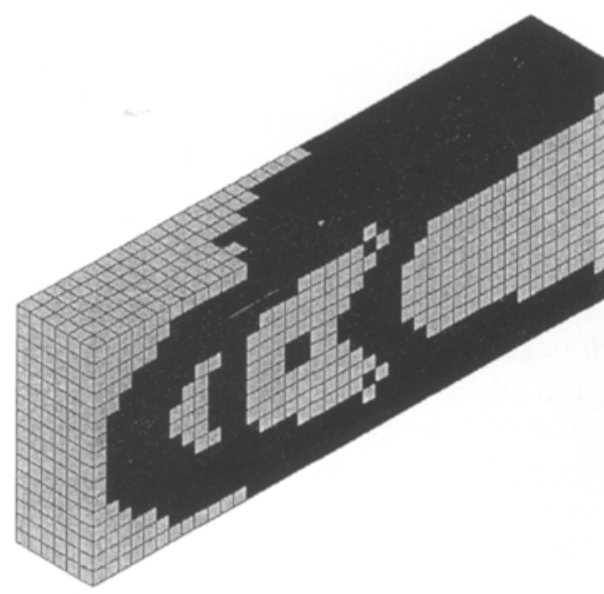

$\mathrm{X}=3$

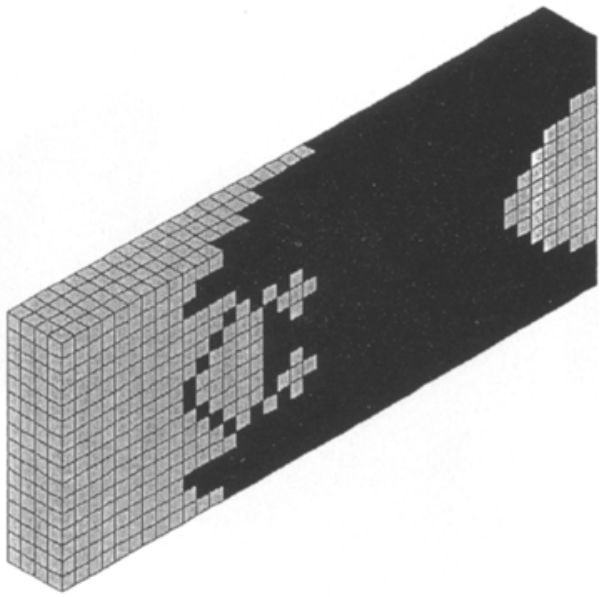

$X=2$

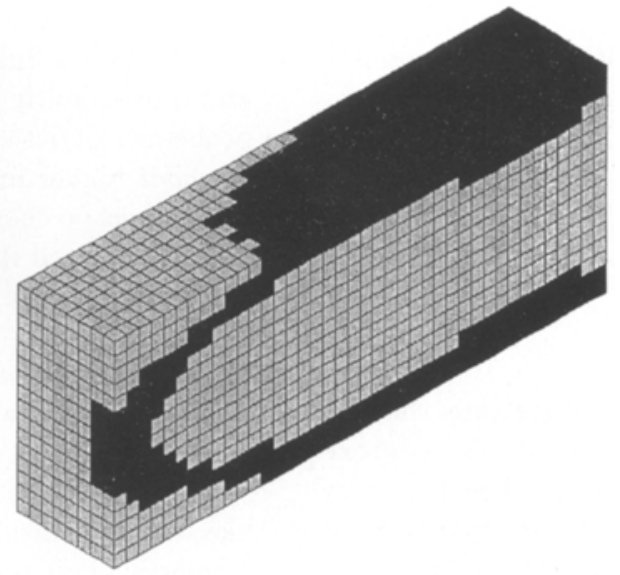

$X=4$

Fig. 11. Final topology for $E^{1} /\left(\max E^{2}\right)=10^{-7}$ (longitudinal cuts)

removed from the figure.

We note here the big gap between the first $\left(10^{-7}\right)$ and second material ratio $\left(10^{-7}\right)$ used in the examples. This is due to the fact that the changes in topology as a function of the material ratio are only easily detectable for ratios above $10^{-2}$.

\subsection{Example 3: Embossed ribs in stamped plates}

The design model described in this work has many applications, among them, the optimum design of reinforcing embossed ribs (also known as beads) in stamped plates. A particular case of the optimization problem (13) is obtained when the local structure of the material " 2 " is fixed and orthotropic, and when material "1" is isotropic. In such cases it is possible to determine the optimum layout of embossed ribs (material "2") within the flat plate (material "1"). In order to solve this particular optimization problem the only modification required is to fix the form of $E^{2}$ in the most inner maximization problem in (13).

Due to the geometric complexity of this real life example it was solved using software developed at Ford for this particular application of optimum layout of embossed ribs of constant properties. The structure in question is the decklid of a sedan vehicle (see Fig. 17) subject to a vertical load at the key switch. The objective of the problem is to maximize the stiffness for such load condition by embossing ribs in the decklid inner panel. The resource constraint metric is the area allowed to be covered by embossed ribs. For this example, embossed areas are limited to $30 \%$ of the total decklid area. Figures 18 and 19 show the "optimum topology" and the "optimum orientation of the principal directions" of the embossed ribs, respectively.

The stiffness of the decklid was increased $100 \%$ with the resulting topology/orientation of ribs.

\section{Discussion}

To contrast the present treatment of two-component composite design with common models for the design of continuum structures based on homogenization interpretation of a twophase material [the subject is surveyed by Bendsøe (1995); see also Cherkaev and Gibiansky (1997) and Lipton (1988)], note 

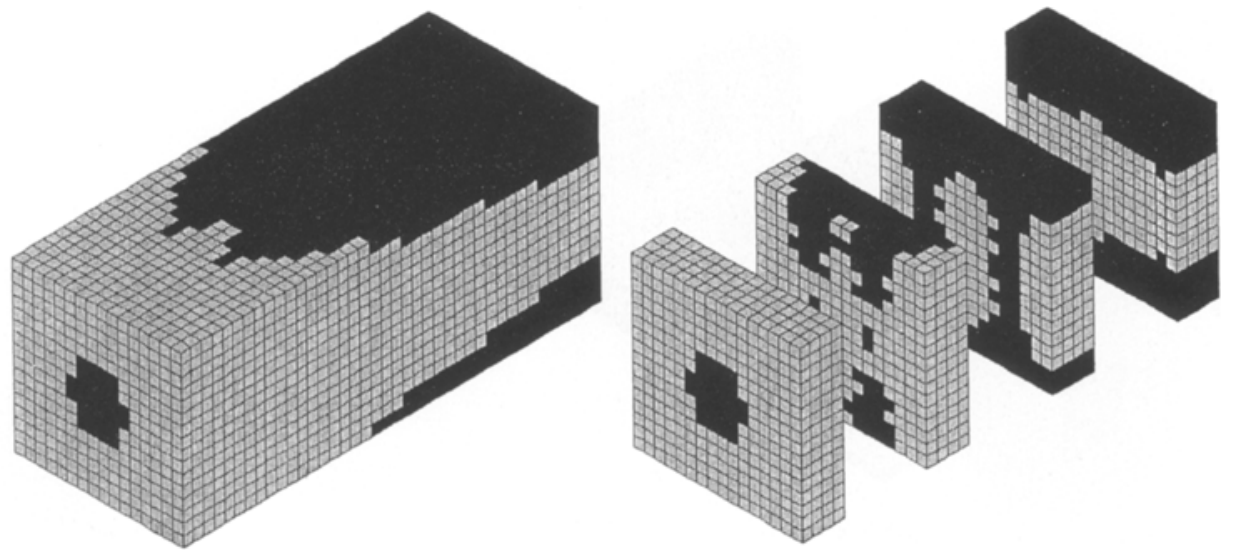

Fig. 12. Final topology for $E^{1} /\left(\max E^{2}\right)=1 / 10$

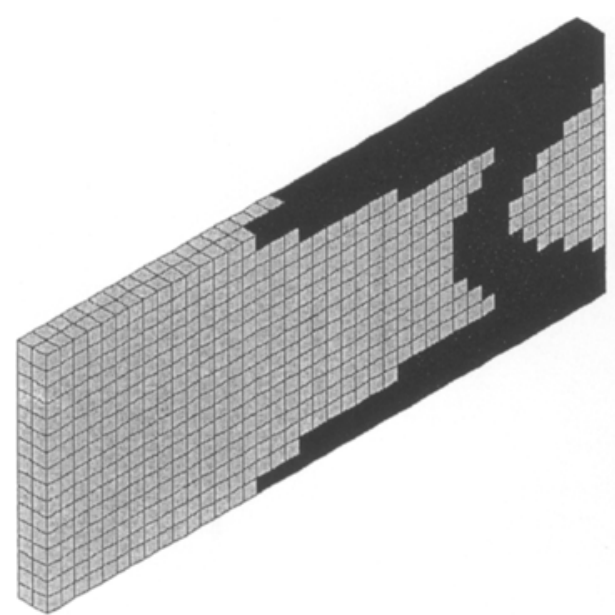

$\mathrm{X}=1$

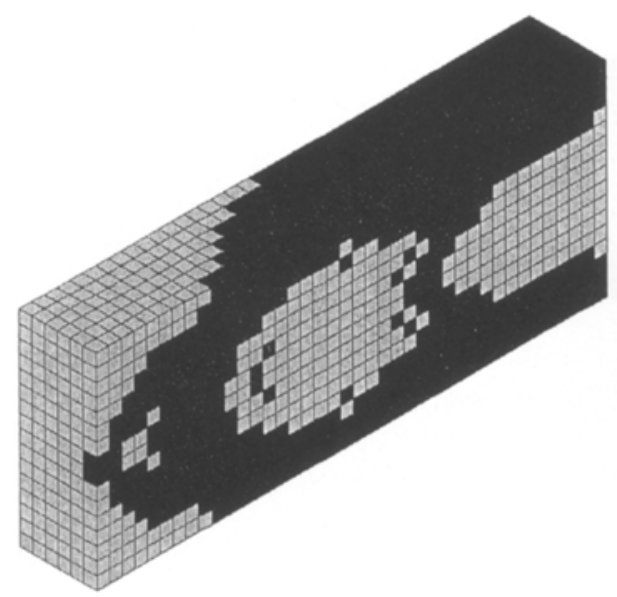

$\mathrm{X}=3$

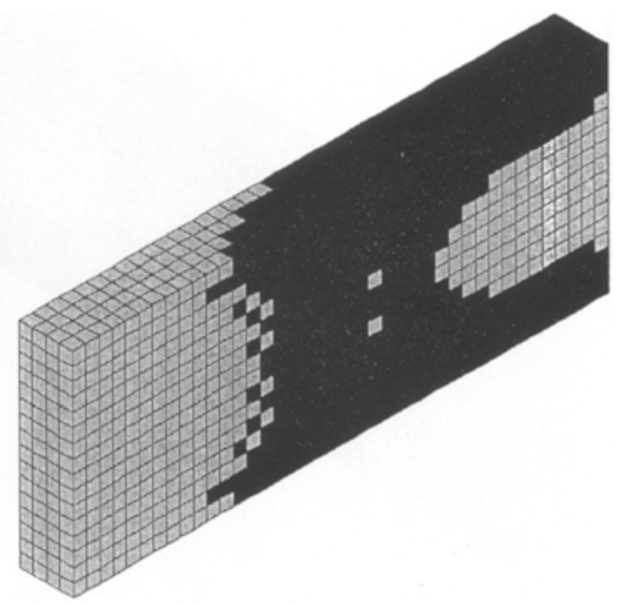

$X=2$

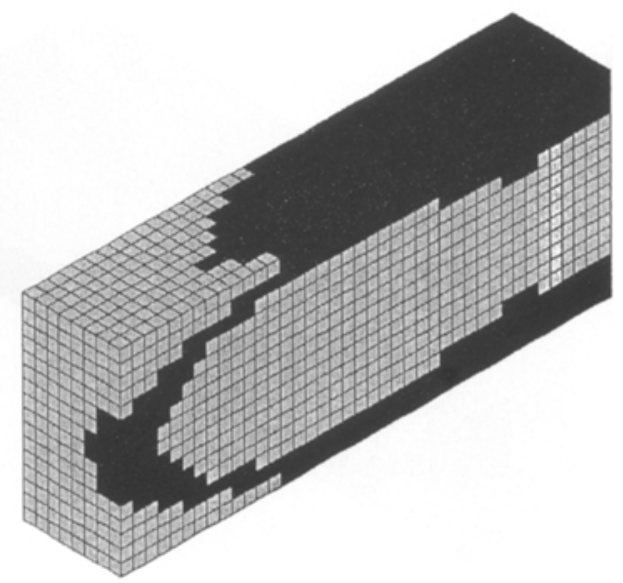

$X=4$

Fig. 13. Final topology for $E^{1} /\left(\max E^{2}\right)=1 / 10$ (longitudinal cuts)

that in the latter the local properties are designated to have specific form, e.g. isotropic, rather than to be represented by a free modulus tensor. Also, the "zero-one" interpretation for the prediction of optimal topology from the latter mod- els [Rozvany et al. (1995) provides a survey on methods for topology design] results in a ill-posed problem, whereas in the present approach topology is predicted on the basis of an ordered sequence of solutions to a well-posed optimization 

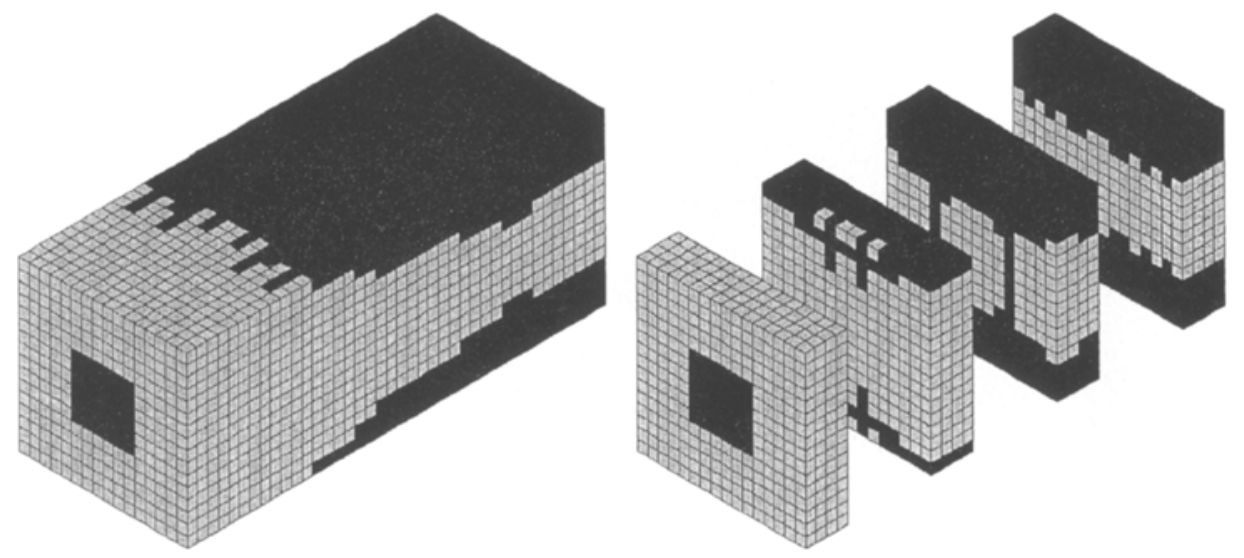

Fig. 14. Final topology for $E^{1} /\left(\max E^{2}\right)=1 / 3$

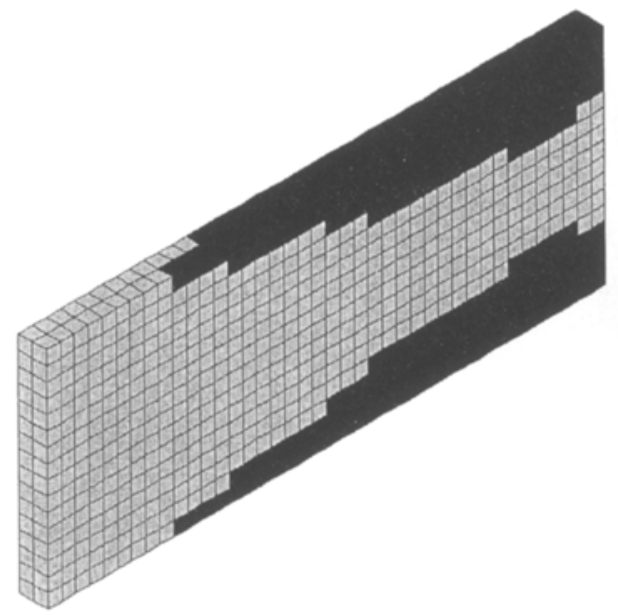

$\mathrm{X}=1$

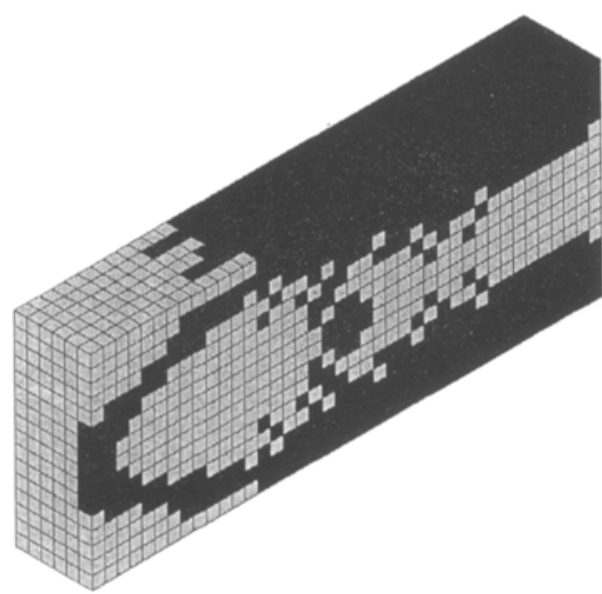

$\mathrm{X}=3$

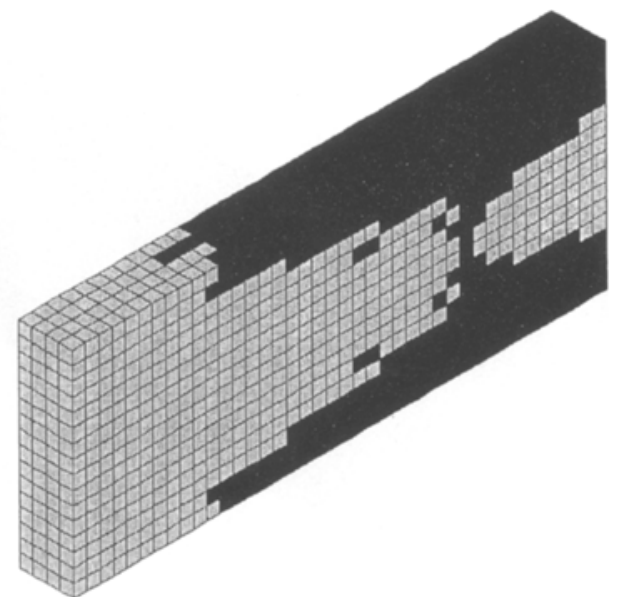

$X=2$

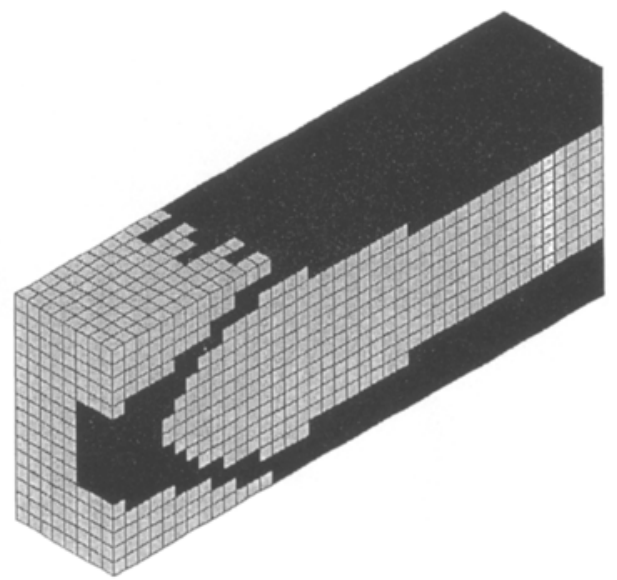

$X=4$

Fig. 15. Final topology for $E^{1} /\left(\max E^{2}\right)=1 / 3$ (longitudinal cuts)

problem.

For simplicity, the model for two-component composite design is described in this paper in a relatively narrow form. However, the same method may be applied equally well in other contexts - for structural design with nonlinear materials as represented by Bendsøe et al. (1996), for example, or for problems where the local measure of material cost is expressed by an invariant other than the trace of the modulus 

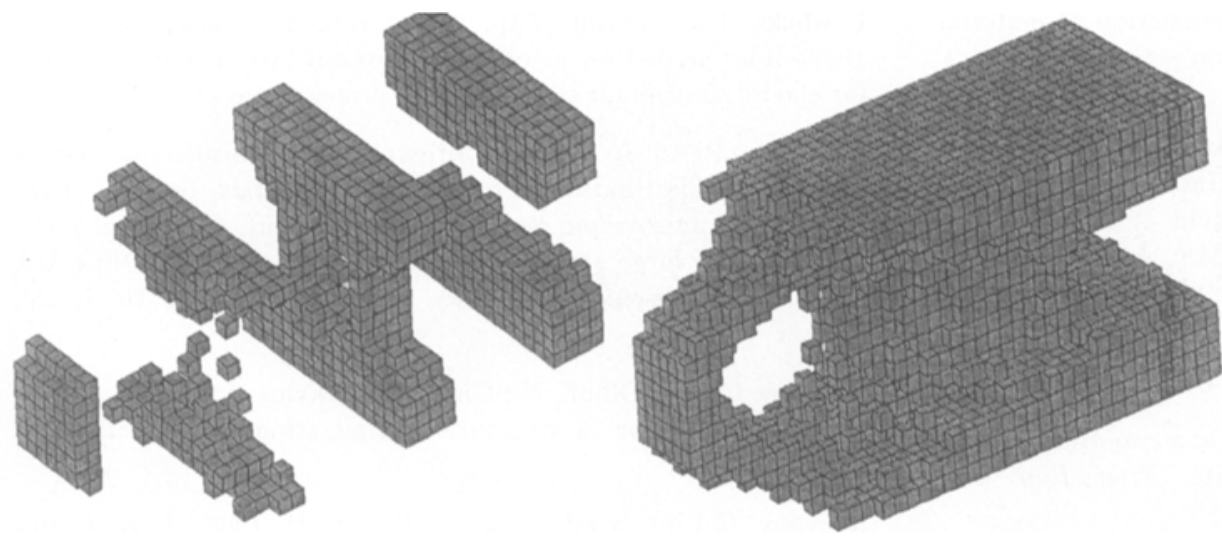

Fig. 16. Final topology for $E^{1} /\left(\max E^{2}\right)=1 / 3$ (material one not shown)

tensor [see e.g. Taylor and Washabaugh (1995) and Taylor (1998) for expressions of generalized cost].

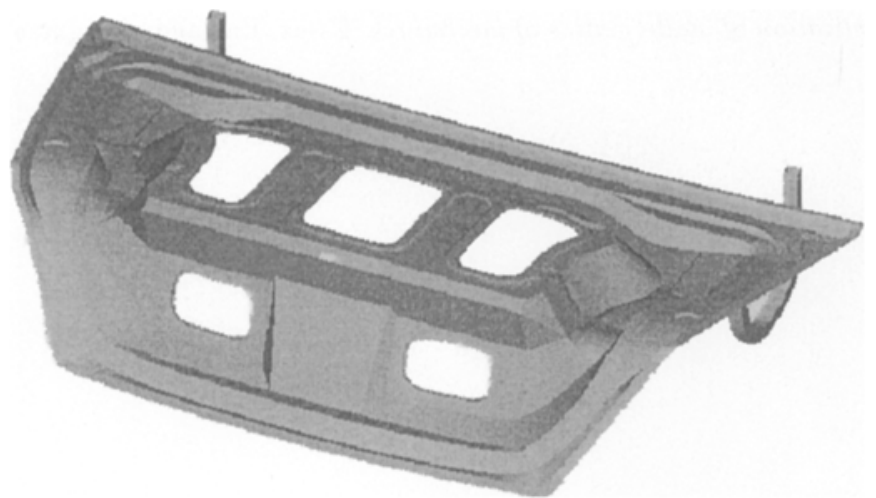

Fig. 17. Computer model of the car decklid

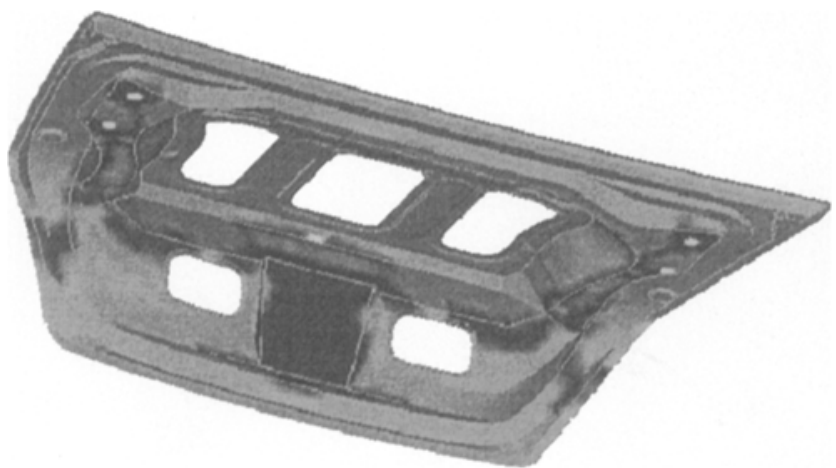

Fig. 18. Optimum topology of embossed ribs using $30 \%$ of the area

\section{Acknowledgements}

The first and third authors express their appreciation for support received from the Ford Motor Company - Scientific Research Laboratories under grant \# 95-106R.

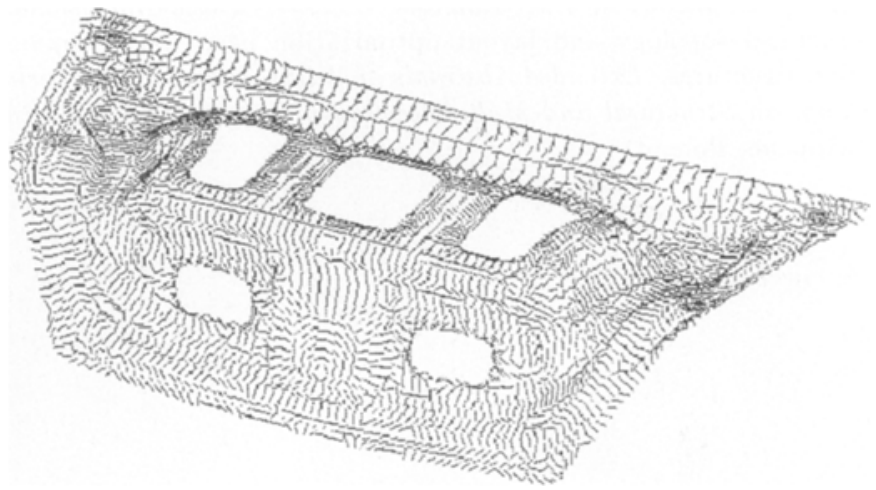

Fig. 19. Optimum orientation of embossed beads

The first author also expresses appreciation for partial support received from INVOTAN through the NATO Science Fellowships Programme.

\section{References}

Bendsøe, M.P. 1995: Optimization of structural topology, shape and material. Berlin, Heidelberg, New York: Springer

Bendsøe, M.P.; Diaz, A.; Lipton, R.; Taylor, J.E. 1995: Optimal design of material properties and material distribution for multiple loading conditions. Int. J. Num. Meth. Engrg. 38, 1149-1170

Bendsøe, M.P.; Guedes, J.M.; Haber, R.B.; Pedersen, P.; Taylor, J.E. 1994: An analytical model to predict optimal material properties in the context of optimal structural design. J. Appl. Mech. $61,930-937$

Bendsøe, M.P.; Guedes, J.M.; Plaxton, S.; Taylor, J.E. 1996: Optimization of structure and material properties for solids composed of softening materials. Int. J. Solids \& Struct. 33, 1799-1813

Cherkaev, A.; Gibiansky, L. 1993: Coupled estimates for the bulk and shear moduli of a two dimensional isotropic elastic composite. J. Mech. \& Physics of Solids 41, 937-980

Diaz, A.; Lipton, R.; Ciro A. Soto 1995: A new formulation of the problem of optimum reinforcement of Reissner-Mindlin plates. Comput. Meth. Appl. Mech. Engng. 123, 121-139 
Guedes, J.M.; Taylor, J.E. 1997a: On the prediction of material properties and topology for optimal continuum structures. Struct. Optim. 14, 193-199

Guedes, J.M.; Taylor J.E. 1997b: An alternative approach for the prediction of optimal structural topology. In: Proc. $M C N U$ '97 Joint ASME, ASCE, SES Summer Mtg. (held at Northwestern University). AMD-Vol. 227. New York: ASME

Jacobs, C.; Simo, J.C.; Beaupre, G.S.; Carter, D.R. 1997: Adaptative bone remodeling incorporating simultaneous density and anisotropy conditions. J. Biomech. 30, 603-613

Lipton, R.P. 1988: On the effective elasticity of a two-dimensional, homogenized incompressible elastic composite. Proc. Roy. Soc. Edin. 109A

Olhoff, N.; Thomsen, J.; Rasmussen, J. 1993: Topology optimization of bi-material structures. In: Pedersen, P. (ed.) Optimal design with advanced materials. Amsterdam: Elsevier

Olhoff, N.; Jacobsen, J.B.; Ronholt, E. 1997: Three-dimensional structural topology and layout optimization based on optimum microstructures. Extended Abstracts of WCSMO-2, Second World Cong. on Structural and Multidisciplinary Optimization (held in Zakopane, Poland)

Received April 22, 1998
Pawlicki, J.R.; Taylor, J.E.; Turteltaub, S.; Washabaugh, P.P. 1998: The prediction of optimal material layout and properties for elastic continum structures. In preparation

Pedersen, P. 1993: Optimal orientation of anisotropic materials/optimal distribution of anisotropic materials, optimal shape design with anisotropic materials. In: Rozvany, G.I.N. (ed.) $O p$ timization of large structural systems (Proc. NATO/DFG ASI, held in Berchtesgaden, Germany, 1991), pp. 649-689 Dordrecht: Kluwer

Rozvany, G.I.N.; Olhoff, N.; Cheng, G.; Taylor, J.E. 1982: On the solid plate paradox in structural optimization. J. Struct. Mech. $10,1-32$

Rozvany, G.I.N.; Bendsøe, M.P.; Kirsch, U. 1995: Layout optimization of structures. Appl. Mech. Rev. 48, 41-119

Taylor, J.E. 1998: An energy modal for the optimal design of continuum structures. Struct. Optim. 16, 116-127

Taylor, J.E.; Washabaugh, P.D. 1995: A generalized expression of cost for prediction of the optimal material properties tensor. In: Manuel, D.P.; Marques, M.; Rodrigues, J.F. (eds.) Trends in application of mathematics of mechanics. Essex, England: Longman 